\title{
New Fixed Point Results with PPF Dependence in Banach Spaces Endowed with a Graph
}

\author{
N. Hussain, ${ }^{1}$ S. Khaleghizadeh, ${ }^{2}$ P. Salimi, ${ }^{3}$ and F. Akbar ${ }^{4}$ \\ ${ }^{1}$ Department of Mathematics, King Abdulaziz University, P.O. Box 80203, Jeddah 21589, Saudi Arabia \\ ${ }^{2}$ Department of Mathematics, Payame Noor University, P.O. Box 19395-3697, Tehran, Iran \\ ${ }^{3}$ Young Researchers and Elite Club, Rasht Branch, Islamic Azad University, Rasht, Iran \\ ${ }^{4}$ Department of Mathematics, GDCW, Bosan Road, Multan, Pakistan
}

Correspondence should be addressed to P. Salimi; salimipeyman@gmail.com

Received 30 October 2013; Accepted 21 November 2013

Academic Editor: Mohammad Mursaleen

Copyright (C) 2013 N. Hussain et al. This is an open access article distributed under the Creative Commons Attribution License, which permits unrestricted use, distribution, and reproduction in any medium, provided the original work is properly cited.

\begin{abstract}
We introduce the concept of an $\alpha_{c}$-admissible non-self-mappings with respect to $\eta_{c}$ and establish the existence of PPF dependent fixed and coincidence point theorems for $\alpha_{c} \eta_{c}-\psi$-contractive non-self-mappings in the Razumikhin class. As applications of our PPF dependent fixed point and coincidence point theorems, we derive some new fixed and coincidence point results for $\psi$-contractions whenever the range space is endowed with a graph or with a partial order. The obtained results generalize, extend, and modify some PPF dependent fixed point results in the literature. Several interesting consequences of our theorems are also provided.
\end{abstract}

\section{Introduction and Preliminaries}

In nonlinear functional analysis, one of the most significant research areas is fixed point theory. On the other hand, fixed point theory has an application in distinct branches of mathematics and also in different sciences, such as engineering, computer science, and economics. In 1922, Banach proved that every contraction in a complete metric space has a unique fixed point. This celebrated result have been generalized and improved by many authors in the context of different abstract spaces for various operators (see [1-31] and references therein). In 1997, Bernfeld et al. [5] introduced the concept of fixed point for mappings that have different domains and ranges, which is called PPF dependent fixed point or the fixed point with PPF dependence. Furthermore, they gave the notion of Banach type contraction for non-selfmapping and also proved the existence of PPF dependent fixed point theorems in the Razumikhin class for Banach type contractions (see [17]). The PPF dependent fixed point theorems are useful for proving the solutions of nonlinear functional differential and integral equations which may depend upon the past history, present data, and future consideration (see [9]). On the other hand, Samet et al. [22] introduced the concept of $\alpha$-admissible self-mappings and proved fixed point results for $\alpha$-admissible contractive mappings in complete metric spaces and provided application of the obtained results to ordinary differential equations. More recently, Salimi et al. [24] modified the notions of $\alpha$ $\psi$-contractive and $\alpha$-admissible mappings and established fixed point theorems to generalize the results in [22]. In this paper, we introduce the concept of an $\alpha_{c}$-admissible nonself-mapping with respect to $\eta_{c}$ and establish the existence of PPF dependent fixed and coincidence point theorems for $\alpha_{c} \eta_{c}-\psi$-contractive non-self-mappings in the Razumikhin class. As applications of our PPF dependent fixed point and coincidence point theorems, we derive some new fixed and coincidence point results for $\psi$-contractions whenever the range space is endowed with a graph or with a partial order. The obtained results generalize, extend, and modify some PPF dependent fixed results in the literature. Several interesting consequences of our theorems are also provided.

Throughout this paper, we assume that $\left(E,\|\cdot\|_{E}\right)$ is a Banach space, $I$ denotes a closed interval $[a, b]$ in $\mathbb{R}$, and 
$E_{0}=C(I, E)$ denotes the set of all continuous $E$-valued functions on $I$ equipped with the supremum norm $\|\cdot\|_{E_{0}}$ defined by

$$
\|\phi\|_{E_{0}}=\sup _{t \in I}\|\phi(t)\|_{E}
$$

For a fixed element $c \in I$, the Razumikhin or minimal class of functions in $E_{0}$ is defined by

$$
\mathscr{R}_{c}=\left\{\phi \in E_{0}:\|\phi\|_{E_{0}}=\|\phi(c)\|_{E}\right\} .
$$

Clearly, every constant function from $I$ to $E$ belongs to $\mathscr{R}_{c}$.

Definition 1. Let $\mathscr{R}_{c}$ be the Razumikhin class, then

(i) the class $\mathscr{R}_{c}$ is algebraically closed with respect to difference, if $\phi-\xi \in \mathscr{R}_{c}$ whenever $\phi, \xi \in \mathscr{R}_{c}$;

(ii) the class $\mathscr{R}_{c}$ is topologically closed if it is closed with respect to the topology on $E_{0}$ generated by the norm $\|\cdot\|_{E_{0}}$.

Definition 2 (see [5]). A mapping $\phi \in E_{0}$ is said to be a PPF dependent fixed point or a fixed point with PPF dependence of mapping $T: E_{0} \rightarrow E$ if $T \phi=\phi(c)$ for some $c \in I$.

Definition 3 (see [17]). Let $S: E_{0} \rightarrow E_{0}$ and let $T: E_{0} \rightarrow E$. A point $\phi \in E_{0}$ is said to be a PPF dependent coincidence point or a coincidence point with PPF dependence of $S$ and $T$ if $T \phi=(S \phi)(c)$ for some $c \in I$.

Definition 4 (see [5]). The mapping $T: E_{0} \rightarrow E$ is called a Banach type contraction if there exists $k \in[0,1)$ such that

$$
\|T \phi-T \xi\|_{E} \leq k\|\phi-\xi\|_{E_{0}}
$$

for all $\phi, \xi \in E_{0}$.

Samet et al. [22] defined the notion of $\alpha$-admissible mappings as follows.

Definition 5. Let $T$ be a self-mapping on $X$ and let $\alpha: X \times$ $X \rightarrow[0,+\infty)$ be a function. We say that $T$ is an $\alpha$-admissible mapping if

$$
x, y \in X, \quad \alpha(x, y) \geq 1 \Longrightarrow \alpha(T x, T y) \geq 1 .
$$

In [22] the authors considered the family $\Psi$ of nondecreasing functions $\psi:[0,+\infty) \rightarrow[0,+\infty)$ such that $\sum_{n=1}^{+\infty} \psi^{n}(t)<+\infty$ for each $t>0$, where $\psi^{n}$ is the $n$th iterate of $\psi$.

Salimi et al. [24] modified and generalized the notions of $\alpha$ - $\psi$-contractive mappings and $\alpha$-admissible mappings as follows.

Definition 6 (see [24]). Let $T$ be a self-mapping on $X$ and $\alpha$, $\eta: X \times X \rightarrow[0,+\infty)$ be two functions. We say that $T$ is an $\alpha$-admissible mapping with respect to $\eta$ if

$$
\begin{array}{r}
\alpha(x, y) \geq \eta(x, y) \Longrightarrow \alpha(T x, T y) \geq \eta(T x, T y), \\
x, y \in X .
\end{array}
$$

Note that if we take $\eta(x, y)=1$, then this definition reduces to Definition 5. Also, if we take, $\alpha(x, y)=1$, then we say that $T$ is an $\eta$-subadmissible mapping.

The following result is a proper generalization of the above-mentioned results.

Theorem 7 (see [24]). Let $(X, d)$ be a complete metric space and let $T$ be an $\alpha$-admissible mapping. Assume that

$$
x, y \in X, \quad \alpha(x, y) \geq 1 \Longrightarrow d(T x, T y) \leq \psi(M(x, y)),
$$

where $\psi \in \Psi$ and

$$
\begin{gathered}
M(x, y)=\max \left\{d(x, y), \frac{d(x, T x)+d(y, T y)}{2},\right. \\
\left.\frac{d(x, T y)+d(y, T x)}{2}\right\} .
\end{gathered}
$$

Also, suppose that the following assertions hold:

(i) there exists $x_{0} \in X$ such that $\alpha\left(x_{0}, T x_{0}\right) \geq 1$,

(ii) either $T$ is continuous or for any sequence $\left\{x_{n}\right\}$ in $X$ with $\alpha\left(x_{n}, x_{n+1}\right) \geq 1$ for all $n \in \mathbb{N} \cup\{0\}$ and $x_{n} \rightarrow x$ as $n \rightarrow+\infty$, we have $\alpha\left(x_{n}, x\right) \geq 1$ for all $n \in \mathbb{N} \cup\{0\}$.

Then $T$ has a fixed point.

For more details on modified $\alpha-\psi$-contractive mappings and related fixed point results we refer the reader to $[8,13,14$, $25,26]$.

\section{PPF Dependent Fixed and Coincidence Point Results}

First we define the notion of non-self $\alpha$-admissible mapping with respect to $\eta$ as follows.

Definition 8. Let $c \in I$ and let $T: E_{0} \rightarrow E, \alpha, \eta: E \times E \rightarrow$ $[0, \infty)$. We say that $T$ is an $\alpha_{c}$-admissible non-self-mapping with respect to $\eta_{c}$ if for $\phi, \xi \in E_{0}$,

$$
\begin{array}{r}
\alpha(\phi(c), \xi(c)) \geq \eta(\phi(c), \xi(c)) \\
\Longrightarrow \alpha(T \phi, T \xi) \geq \eta(T \phi, T \xi)
\end{array}
$$

Note that if we take $\eta(\phi(c), \xi(c))=1$, then we say $T$ is an $\alpha_{c}$ admissible non-self-mapping. Also, if we take $\alpha(\phi(c), \xi(c))=$ 1 , then we say that $T$ is an $\eta_{c}$-subadmissible non-selfmapping.

Example 9. Let $E=\mathbb{R}$ be a real Banach space with usual norm and let $I=[0,1]$. Define $T: E_{0} \rightarrow E$ by $T \phi=(1 / 2) \phi(1)$ for all $\phi \in E_{0}$ and $\alpha, \eta: E \times E \rightarrow[0,+\infty)$ by

$$
\alpha(x, y)= \begin{cases}x^{4}+y^{8}+1, & \text { if } x \geq y \\ \frac{1}{3}, & \text { otherwise }\end{cases}
$$


$\eta(x, y)=x^{4}+1 / 2$. Then, $T$ is an $\alpha_{1}$-admissible mapping with respect to $\eta_{1}$. In fact, if $\alpha(\phi(1), \xi(1)) \geq \eta(\phi(1), \xi(1))$, then $\phi(1) \geq \xi(1)$ and so, $(1 / 2) \phi(1) \geq(1 / 2) \xi(1)$. That is, $T \phi \geq T \xi$ which implies that $\alpha(T \phi, T \xi) \geq \eta(T \phi, T \xi)$.

Denote with $\Psi$ the family of nondecreasing functions $\psi$ : $[0,+\infty) \rightarrow[0,+\infty)$ such that $\sum_{n=1}^{\infty} \psi^{n}(t)<+\infty$ for all $t>0$, where $\psi^{n}$ is the $n$th iterate of $\psi$.

The following Remark is obvious.

Remark 10. If $\psi \in \Psi$, then $\psi(t)<t$ for all $t>0$.

Definition 11. Let $T: E_{0} \rightarrow E, \alpha, \eta: E \times E \rightarrow[0, \infty)$ be three mappings and $c \in I$. Then,

(i) $T$ is an $\alpha_{c} \eta_{c}-\psi$-contractive non-self-mapping if

$$
\begin{aligned}
& \alpha(\phi(c), \xi(c)) \geq \eta(\phi(c), T \phi) \\
& \quad \Longrightarrow\|T \phi-T \xi\|_{E} \leq \psi(M(\phi, \xi)),
\end{aligned}
$$

(ii) $T$ is a modified $\alpha_{c}-\psi$-contractive non-self-mapping if $\alpha(\phi(c), \xi(c)) \geq 1 \Longrightarrow\|T \phi-T \xi\|_{E} \leq \psi(M(\phi, \xi))$,

where $\psi \in \Psi$ and

$$
\begin{gathered}
M(\phi, \xi) \\
=\max \left\{\|\phi-\xi\|_{E_{0}}, \frac{\|\phi(c)-T \phi\|_{E}+\|\xi(c)-T \xi\|_{E}}{2},\right. \\
\left.\frac{\|\phi(c)-T \xi\|_{E}+\|\xi(c)-T \phi\|_{E}}{2}\right\} .
\end{gathered}
$$

The following theorem is our first main result in this section.

Theorem 12. Let $T: E_{0} \rightarrow E, \alpha, \eta: E \times E \rightarrow[0, \infty)$ be three mappings that satisfy the following assertions:

(i) there exists $c \in I$ such that $\mathscr{R}_{c}$ is topologically closed and algebraically closed with respect to difference;

(ii) $T$ is an $\alpha_{c}$-admissible non-self-mapping with respect to $\eta_{c}$

(iii) $T$ is an $\alpha_{c} \eta_{c}-\psi$-contractive non-self-mapping;

(iv) if $\left\{\phi_{n}\right\}$ is a sequence in $E_{0}$ such that $\phi_{n} \rightarrow \phi$ as $n \rightarrow \infty$ and $\alpha\left(\phi_{n}(c), \phi_{n+1}(c)\right) \geq \eta\left(\phi_{n}(c), \phi_{n+1}(c)\right)$ for all $n \in$ $\mathbb{N} \cup\{0\}$, then $\alpha\left(\phi_{n}(c), \phi(c)\right) \geq \eta\left(\phi_{n}(c), T \phi_{n}\right)$ for all $n \in$ $\mathbb{N} \cup\{0\} ;$

(v) there exists $\phi_{0} \in \mathscr{R}_{c}$ such that $\alpha\left(\phi_{0}(c), T \phi_{0}\right) \geq$ $\eta\left(\phi_{0}(c), T \phi_{0}\right)$.

Then, $T$ has a PPF dependent fixed point.

Proof. Let, $\phi_{0} \in \mathscr{R}_{c}$. Since $T \phi_{0} \in E$, there exists $x_{1} \in E$ such that $T \phi_{0}=x_{1}$. Choose $\phi_{1} \in \mathscr{R}_{c}$ such that,

$$
x_{1}=\phi_{1}(c) .
$$

By continuing this process, by induction, we can build a sequence $\left\{\phi_{n}\right\}$ in $\mathscr{R}_{c} \subseteq E_{0}$ such that

$$
T \phi_{n-1}=\phi_{n}(c), \quad \forall n \in \mathbb{N} .
$$

Since $\mathscr{R}_{c}$ is algebraically closed with respect to difference, it follows that

$$
\left\|\phi_{n-1}-\phi_{n}\right\|_{E_{0}}=\left\|\phi_{n-1}(c)-\phi_{n}(c)\right\|_{E}, \quad \forall n \in \mathbb{N} .
$$

If there exists $n_{0} \in \mathbb{N}$ such that $\phi_{n_{0}}(c)=\phi_{n_{0}+1}(c)=T \phi_{n_{0}}$, then $\phi_{n_{0}}$ is a PPF dependent fixed point of $T$ and we have nothing to prove. Hence we assume that $\phi_{n-1} \neq \phi_{n}$ for all $n \in \mathbb{N}$.

Since $T$ is an $\alpha_{c}$-admissible non-self-mapping with respect to $\eta_{c}$ and

$$
\begin{aligned}
\alpha\left(\phi_{0}(c), \phi_{1}(c)\right) & =\alpha\left(\phi_{0}(c), T \phi_{0}\right) \\
& \geq \eta\left(\phi_{0}(c), T \phi_{0}\right)=\eta\left(\phi_{0}(c), \phi_{1}(c)\right),
\end{aligned}
$$

so,

$$
\alpha\left(\phi_{1}(c), T \phi_{1}\right) \geq \eta\left(\phi_{1}(c), T \phi_{1}\right) .
$$

By continuing this process we get

$$
\begin{aligned}
\alpha\left(\phi_{n-1}(c), \phi_{n}(c)\right) & =\alpha\left(\phi_{n-1}(c), T \phi_{n-1}\right) \\
& \geq \eta\left(\phi_{n-1}(c), T \phi_{n-1}\right),
\end{aligned}
$$

for all $n \in \mathbb{N}$. Then from (10) we get

$$
\begin{aligned}
\left\|\phi_{n}-\phi_{n+1}\right\|_{E_{0}} & =\left\|\phi_{n}(c)-\phi_{n+1}(c)\right\|_{E} \\
& =\left\|T \phi_{n-1}-T \phi_{n}\right\|_{E} \leq \psi\left(M\left(\phi_{n-1}, \phi_{n}\right)\right),
\end{aligned}
$$

where

$$
\begin{aligned}
M\left(\phi_{n-1}, \phi_{n}\right) & \\
=\max & \left\{\left\|\phi_{n-1}-\phi_{n}\right\|_{E_{0}},\right. \\
& \frac{\left\|\phi_{n-1}(c)-T \phi_{n-1}\right\|_{E}+\left\|\phi_{n}(c)-T \phi_{n}\right\|_{E}}{2}, \\
& \left.\frac{\left\|\phi_{n-1}(c)-T \phi_{n}\right\|_{E}+\left\|\phi_{n}(c)-T \phi_{n-1}\right\|_{E}}{2}\right\} \\
=\max & \left\{\left\|\phi_{n-1}-\phi_{n}\right\|_{E_{0}},\right. \\
& \frac{\left\|\phi_{n-1}(c)-\phi_{n}(c)\right\|_{E}+\left\|\phi_{n}(c)-\phi_{n+1}(c)\right\|_{E}}{2}, \\
& \left.\frac{\left\|\phi_{n-1}(c)-\phi_{n+1}(c)\right\|_{E}+\left\|\phi_{n}(c)-\phi_{n}(c)\right\|_{E}}{2}\right\}
\end{aligned}
$$




$$
\begin{gathered}
=\max \left\{\left\|\phi_{n-1}-\phi_{n}\right\|_{E_{0}},\right. \\
\frac{\left\|\phi_{n-1}-\phi_{n}\right\|_{E_{0}}+\left\|\phi_{n}-\phi_{n+1}\right\|_{E_{0}}}{2}, \\
\left.\frac{\left.\left\|\phi_{n-1}-\phi_{n+1}\right\|_{E_{0}}\right\}}{2}\right\} \\
\leq \max \left\{\left\|\phi_{n-1}-\phi_{n}\right\|_{E_{0}},\right. \\
\left.\frac{\left\|\phi_{n-1}-\phi_{n}\right\|_{E_{0}}+\left\|\phi_{n}-\phi_{n+1}\right\|_{E_{0}}}{2}\right\} \\
\leq \max \left\{\left\|\phi_{n-1}-\phi_{n}\right\|_{E_{0}},\left\|\phi_{n}-\phi_{n+1}\right\|_{E_{0}}\right\}
\end{gathered}
$$

which implies that

$$
\begin{aligned}
& \left\|\phi_{n}-\phi_{n+1}\right\|_{E_{0}} \\
& \quad \leq \psi\left(\max \left\{\left\|\phi_{n-1}-\phi_{n}\right\|_{E_{0}},\left\|\phi_{n}-\phi_{n+1}\right\|_{E_{0}}\right\}\right) .
\end{aligned}
$$

Now, if $\max \left\{\left\|\phi_{n-1}-\phi_{n}\right\|_{E_{0}},\left\|\phi_{n}-\phi_{n+1}\right\|_{E_{0}}\right\}=\left\|\phi_{n}-\phi_{n+1}\right\|_{E_{0}}$, then

$$
\left\|\phi_{n}-\phi_{n+1}\right\|_{E_{0}} \leq \psi\left(\left\|\phi_{n}-\phi_{n+1}\right\|_{E_{0}}\right)<\left\|\phi_{n}-\phi_{n+1}\right\|_{E_{0}}
$$

which is a contradiction. Hence,

$$
\left\|\phi_{n}-\phi_{n+1}\right\|_{E_{0}} \leq \psi\left(\left\|\phi_{n-1}-\phi_{n}\right\|_{E_{0}}\right)
$$

for all $n \in \mathbb{N}$. So,

$$
\left\|\phi_{n}-\phi_{n+1}\right\|_{E_{0}} \leq \psi^{n}\left(\left\|\phi_{0}-\phi_{1}\right\|_{E_{0}}\right),
$$

for all $n \in \mathbb{N}$.

Fix $\epsilon>0$, then there exists $N \in \mathbb{N}$ such that

$$
\sum_{n \geq N} \psi^{n}\left(\left\|\phi_{0}-\phi_{1}\right\|_{E_{0}}\right)<\epsilon \quad \forall n \in \mathbb{N} .
$$

Let $m, n \in \mathbb{N}$ with $m>n \geq N$. By triangular inequality we get

$$
\begin{aligned}
\left\|\phi_{n}-\phi_{m}\right\|_{E_{0}} & \leq \sum_{k=n}^{m-1}\left\|\phi_{k}-\phi_{k+1}\right\|_{E_{0}} \\
& \leq \sum_{n \geq N} \psi^{n}\left(\left\|\phi_{0}-\phi_{1}\right\|_{E_{0}}\right)<\epsilon .
\end{aligned}
$$

Consequently, $\lim _{m, n \rightarrow+\infty}\left\|\phi_{n}-\phi_{m}\right\|_{E_{0}}=0$. Hence $\left\{\phi_{n}\right\}$ is a Cauchy sequence in $\mathscr{R}_{c} \subseteq E_{0}$. By the completeness of $E_{0},\left\{\phi_{n}\right\}$ converges to a point $\phi^{*} \in E_{0}$, that is, $\phi_{n} \rightarrow \phi^{*}$, as $n \rightarrow \infty$. Since $\mathscr{R}_{c}$ is topologically closed, we deduce that $\phi^{*} \in \mathscr{R}_{c}$.
From (iv) we have $\alpha\left(\phi_{n}(c), \phi(c)\right) \geq \eta\left(\phi_{n}(c), T \phi_{n}\right)$ for all $n \in$ $\mathbb{N} \cup\{0\}$. By (10) we have

$$
\begin{aligned}
\| T \phi^{*} & -\phi^{*}(c) \|_{E} \\
& \leq\left\|T \phi^{*}-T \phi_{n}\right\|_{E}+\left\|T \phi_{n}-\phi^{*}(c)\right\|_{E} \\
& =\left\|T \phi^{*}-T \phi_{n}\right\|_{E}+\left\|\phi_{n+1}(c)-\phi^{*}(c)\right\|_{E} \\
& \leq \psi\left(M\left(\phi^{*}, \phi_{n}\right)\right)+\left\|\phi_{n+1}-\phi^{*}\right\|_{E_{0}} \\
& <M\left(\phi^{*}, \phi_{n}\right)+\left\|\phi_{n+1}-\phi^{*}\right\|_{E_{0}},
\end{aligned}
$$

where

$$
\begin{gathered}
M\left(\phi^{*}, \phi_{n}\right) \\
=\max \left\{\left\|\phi^{*}-\phi_{n}\right\|_{E_{0}},\right. \\
\frac{\left\|\phi^{*}(c)-T \phi^{*}\right\|_{E}+\left\|\phi_{n}(c)-T \phi_{n}\right\|_{E}}{2}, \\
\left.\frac{\left\|\phi^{*}(c)-T \phi_{n}\right\|_{E}+\left\|\phi_{n}(c)-T \phi^{*}\right\|_{E}}{2}\right\} \\
=\max \left\{\left\|\phi^{*}-\phi_{n}\right\|_{E_{0}}, \quad\right. \\
\frac{\left\|\phi^{*}(c)-T \phi^{*}\right\|_{E}+\left\|\phi_{n}(c)-\phi_{n+1}(c)\right\|_{E}}{2}, \\
\left.\frac{\left\|\phi^{*}(c)-\phi_{n+1}(c)\right\|_{E}+\left\|\phi_{n}(c)-T \phi^{*}\right\|_{E}}{2}\right\} .
\end{gathered}
$$

Taking limit as $n \rightarrow \infty$ in the above inequality we get

$$
\left\|T \phi^{*}-\phi^{*}(c)\right\|_{E} \leq \frac{1}{2}\left\|T \phi^{*}-\phi^{*}(c)\right\|_{E} .
$$

Therefore, $\left\|T \phi^{*}-\phi^{*}(c)\right\|_{E}=0$. That is, $T \phi^{*}=\phi^{*}(c)$. This implies that $\phi^{*}$ is a PPF dependent fixed point of $T$ in $\mathscr{R}_{c}$.

If in Theorem 12 we take $\eta(\phi, \xi)=1$ for all $\phi, \xi \in E_{0}$, then we deduce the following corollary.

Corollary 13. Let $T: E_{0} \rightarrow E$ and $\alpha: E \times E \rightarrow[0, \infty)$ be two mappings satisfy that the following assertions:

(i) there exists $c \in I$ such that $\mathscr{R}_{c}$ is topologically closed and algebraically closed with respect to difference;

(ii) $T$ is an $\alpha_{c}$-admissible non-self-mapping;

(iii) $T$ is a modified $\alpha_{c}-\psi$-contractive non-self-mapping;

(iv) if $\left\{\phi_{n}\right\}$ is a sequence in $E_{0}$ such that $\phi_{n} \rightarrow \phi$ as $n \rightarrow \infty$ and $\alpha\left(\phi_{n}(c), \phi_{n+1}(c)\right) \geq 1$ for all $n \in \mathbb{N} \cup\{0\}$, then $\alpha\left(\phi_{n}(c), \phi(c)\right) \geq 1$ for all $n \in \mathbb{N} \cup\{0\} ;$

(v) there exists $\phi_{0} \in \mathscr{R}_{c}$ such that $\alpha\left(\phi_{0}(c), T \phi_{0}\right) \geq 1$.

Then, $T$ has a PPF dependent fixed point.

We now introduce the notion of $\alpha_{c}$-admissible mapping with respect to $\eta_{c}$ for the pair of maps $(S, T)$ as follows. 
Definition 14. Let $c \in I, S: E_{0} \rightarrow E_{0}, T: E_{0} \rightarrow E$ and let $\alpha, \eta: E \times E \rightarrow[0, \infty)$. We say that the pair $(S, T)$ is an $\alpha_{c}$-admissible with respect to $\eta_{c}$, if for $\phi, \xi \in E_{0}$,

$$
\begin{gathered}
\alpha((S \phi)(c),(S \xi)(c)) \geq \eta((S \phi)(c),(S \xi)(c)) \\
\Longrightarrow \alpha(T \phi, T \xi) \geq \eta(T \phi, T \xi) .
\end{gathered}
$$

Note that if we take $\eta((S \phi)(c), S(\xi)(c))=1$, then we say that the pair $(S, T)$ is an $\alpha_{c}$-admissible mapping. Also, if we take $\alpha((S \phi)(c), S(\xi)(c))=1$, then we say that the pair $(S, T)$ is an $\eta_{c}$-subadmissible mapping.

Now we introduce the notion of $\alpha_{c} \eta_{c}-\psi$-contractiveness for the pair $(S, T)$ as follows.

Definition 15. Let $c \in I, S: E_{0} \rightarrow E_{0}, T: E_{0} \rightarrow E$ and $\alpha$, $\eta: E \times E \rightarrow[0, \infty)$. Then,

(i) we say that the pair $(S, T)$ is an $\alpha_{c} \eta_{c}-\psi$-contractive if

$$
\begin{array}{r}
\alpha((S \phi)(c), \xi(c)) \geq \eta((S \phi)(c), T \phi) \\
\Longrightarrow\|T \phi-T \xi\|_{E} \leq \psi(N(\phi, \xi)) ;
\end{array}
$$

(ii) we say that the pair $(S, T)$ is a modified $\alpha_{c}-\psi$ contractive if

$$
\alpha((S \phi)(c), \xi(c)) \geq 1 \Longrightarrow\|T \phi-T \xi\|_{E} \leq \psi(N(\phi, \xi))
$$

where $\psi \in \Psi$ and

$$
\begin{aligned}
N(\phi, \xi) & \\
=\max & \left\{\|S \phi-S \xi\|_{E_{0}},\right. \\
& \frac{\|(S \phi)(c)-T(S \phi)\|_{E}+\|(S \xi)(c)-T(S \xi)\|_{E}}{2}, \\
& \left.\frac{\|(S \phi)(c)-T(S \xi)\|_{E}+\|(S \phi)(c)-T(S \xi)\|_{E}}{2}\right\} .
\end{aligned}
$$

Theorem 16. Let $S: E_{0} \rightarrow E_{0}, T: E_{0} \rightarrow E, \alpha, \eta: E \times E \rightarrow$ $[0, \infty)$ be four mappings satisfying the following assertions:

(i) there exists $c \in I$ such that $S\left(\mathscr{R}_{c}\right)$ is topologically closed and algebraically closed with respect to difference;

(ii) the pair $(S, T)$ is an $\alpha_{c}$-admissible with respect to $\eta_{c}$;

(iii) the pair $(S, T)$ is an $\alpha_{c} \eta_{c}-\psi$-contractive;

(iv) if $\left\{S \phi_{n}\right\}$ is a sequence in $E_{0}$ such that $S \phi_{n} \rightarrow$ $S \phi$ as $n \rightarrow \infty$ and $\alpha\left(\left(S \phi_{n}\right)(c),\left(S \phi_{n+1}\right)(c)\right) \quad \geq$ $\eta\left(\left(S \phi_{n}\right)(c),\left(S \phi_{n+1}\right)(c)\right)$ for all $n \in \mathbb{N} \cup\{0\}$, then $\alpha\left(\left(S \phi_{n}\right)(c), S \phi(c)\right) \geq \eta\left(\left(S \phi_{n}\right)(c), T \phi_{n}\right)$ for all $n \in \mathbb{N} \cup$ $\{0\}$;

(v) there exists $S \phi_{0} \in S\left(\mathscr{R}_{c}\right)$ such that $\alpha\left(S \phi_{0}(c), T \phi_{0}\right) \geq$ $\eta\left(S \phi_{0}(c), T \phi_{0}\right)$

Then, $S$ and T have a PPF dependent coincidence point.
Proof. As $S: E_{0} \rightarrow E_{0}$, so there exists $F_{0} \subseteq E_{0}$ such that $S\left(F_{0}\right)=S\left(E_{0}\right)$ and $\left.S\right|_{F_{0}}$ is one-to-one. Since $T\left(F_{0}\right) \subseteq T\left(E_{0}\right) \subseteq$ $E$, we can define the mapping $\mathscr{A}: S\left(F_{0}\right) \rightarrow E$ by $\mathscr{A}(S \phi)=T \phi$ for all $\phi \in F_{0}$. Since $\left.S\right|_{F_{0}}$ is one-to-one, then $\mathscr{A}$ is well defined. Let

$$
\begin{aligned}
& \alpha((S \phi)(c),(S \xi)(c)) \geq \eta((S \phi)(c), T \phi), \text { then } \\
& \alpha((S \phi)(c),(S \xi)(c)) \geq \eta((S \phi)(c), \mathscr{A}(S \phi)) .
\end{aligned}
$$

Therefore, by (31) we have

$$
\|\mathscr{A}(S \phi)-\mathscr{A}(S \xi)\|_{E} \leq \psi(N(\phi, \xi)),
$$

where

$$
\begin{aligned}
N(\phi, \xi) & \\
=\max & \left\{\|S \phi-S \xi\|_{E_{0}},\right. \\
& \frac{\|(S \phi)(c)-\mathscr{A}(S \phi)\|_{E}+\|(S \xi)(c)-\mathscr{A}(S \xi)\|_{E}}{2}, \\
& \left.\frac{\|(S \phi)(c)-\mathscr{A}(S \xi)\|_{E}+\|(S \xi)(c)-\mathscr{A}(S \phi)\|_{E}}{2}\right\} .
\end{aligned}
$$

This shows that $\mathscr{A}$ is an $\alpha_{c} \eta_{c}-\psi$-contractive non-selfmapping. Further, all other conditions of Theorem 12 hold true for $\mathscr{A}$. Thus, there exists PPF dependent fixed point $\varphi \in$ $S\left(F_{0}\right)$ of $\mathscr{A}$; that is, $\mathscr{A} \varphi=\varphi(c)$. Since $\varphi \in S\left(F_{0}\right)$, so there exists $\omega \in F_{0}$ such that $S \omega=\varphi$. Thus,

$$
T \omega=\mathscr{A}(S \omega)=\mathscr{A} \varphi=\varphi(c)=(S \omega)(c) .
$$

That is, $\omega$ is a PPF dependent coincidence point of $S$ and $T$.

Corollary 17. Let $S: E_{0} \rightarrow E_{0}, T: E_{0} \rightarrow E, \alpha: E \times E \rightarrow$ $[0, \infty)$ be three mappings satisfying the following assertions:

(i) there exists $c \in I$ such that $S\left(\mathscr{R}_{c}\right)$ is topologically closed and algebraically closed with respect to difference;

(ii) the pair $(S, T)$ is an $\alpha_{c}$-admissible;

(iii) the pair $(S, T)$ is a modified $\alpha_{c}-\psi$-contractive;

(iv) if $\left\{S \phi_{n}\right\}$ is a sequence in $E_{0}$ such that $S \phi_{n} \rightarrow S \phi$ as $n \rightarrow \infty$ and $\alpha\left(\left(S \phi_{n}\right)(c),\left(S \phi_{n+1}\right)(c)\right) \geq 1$ for all $n \in$ $\mathbb{N} \cup\{0\}$, then $\alpha\left(\left(S \phi_{n}\right)(c), S \phi(c)\right) \geq 1$ for all $n \in \mathbb{N} \cup\{0\} ;$

(v) there exists $S \phi_{0} \in S\left(\mathscr{R}_{c}\right)$ such that $\alpha\left(S \phi_{0}(c), T \phi_{0}\right) \geq 1$.

Then, $S$ and T have a PPF dependent coincidence point.

\section{Some Results in Banach Spaces Endowed with a Graph}

Consistent with Jachymski [15], let $(E, d)$ be a metric space where $d(x, y)=\|x-y\|_{E}$ for all $x, y \in E$ and $\Delta$ denotes the diagonal of the Cartesian product of $X \times X$. Consider a directed graph $G$ such that the set $V(G)$ of its vertices 
coincides with $X$, and the set $E(G)$ of its edges contains all loops; that is, $E(G) \supseteq \Delta$. We assume that $G$ has no parallel edges, so we can identify $G$ with the pair $(V(G), E(G))$. Moreover, we may treat $G$ as a weighted graph (see [16, page 309]) by assigning to each edge the distance between its vertices. If $x$ and $y$ are vertices in a graph $G$, then a path in $G$ from $x$ to $y$ of length $N(N \in \mathbb{N})$ is a sequence $\left\{x_{i}\right\}_{i=0}^{N}$ of $N+1$ vertices such that $x_{0}=x, x_{N}=y$ and $\left(x_{i-1}, x_{i}\right) \in E(G)$ for $i=1, \ldots, N$. A graph $G$ is connected if there is a path between any two vertices. $G$ is weakly connected if $\widetilde{G}$ is connected (see for more details $[6,11,15])$.

Definition 18 (see [15]). Let $(X, d)$ be a metric space endowed with a graph $G$. We say that a self-mapping $T: X \rightarrow X$ is a Banach $G$-contraction or simply a $G$-contraction if $T$ preserves the edges of $G$; that is,

$$
\forall x, y \in X, \quad(x, y) \in E(G) \Longrightarrow(T x, T y) \in E(G)
$$

and $T$ decreases weights of the edges of $G$ in the following way:

$$
\begin{gathered}
\exists \alpha \in(0,1) \text { such that } \forall x, y \in X, \\
(x, y) \in E(G) \Longrightarrow d(T x, T y) \leq \alpha d(x, y) .
\end{gathered}
$$

Theorem 19. Let $T: E_{0} \rightarrow E$ and $E$ endowed with a graph $G$. Suppose that the following assertions hold true:

(i) there exists $c \in I$ such that $\mathscr{R}_{c}$ is topologically closed and algebraically closed with respect to difference;

(ii) if $(\phi(c), \xi(c)) \in E(G)$, then $(T \phi, T \xi) \in E(G)$;

(iii) assume that

$$
\begin{array}{r}
(\phi(c), \xi(c)) \in E(G) \Longrightarrow\|T \phi-T \xi\|_{E} \leq \psi(M(\phi, \xi)), \\
\phi, \xi \in E_{0}, \quad \text { where } \psi \in \Psi ;
\end{array}
$$

(iv) if $\left\{\phi_{n}\right\}$ is a sequence in $E_{0}$ such that $\phi_{n} \rightarrow \phi$ as $n \rightarrow \infty$ and $\left(\phi_{n}(c), \phi_{n+1}(c)\right) \in E(G)$ for all $n \in \mathbb{N} \cup\{0\}$, then $\left(\phi_{n}(c), \phi(c)\right) \in E(G)$ for all $n \in \mathbb{N} \cup\{0\} ;$

(v) there exists $\phi_{0} \in \mathscr{R}_{c}$ such that $\left(\phi_{0}(c), T \phi_{0}\right) \in E(G)$.

Then, $T$ has a PPF dependent fixed point.

Proof. Define $\alpha: E \times E \rightarrow[0,+\infty)$ by

$$
\alpha(x, y)= \begin{cases}1, & \text { if }(x, y) \in E(G) \\ \frac{1}{2} & \text { otherwise. }\end{cases}
$$

First, we prove that $T$ is an $\alpha_{c}$-admissible non-self-mapping. Assume that $\alpha(\phi(c), \xi(c)) \geq 1$. Then, we have $(\phi(c), \xi(c)) \in$ $E(G)$. From (ii), we have $(T \phi, T \xi) \in E(G)$; that is, $\alpha(T \phi, T \xi) \geq$ 1. Thus $T$ is an $\alpha_{c}$-admissible non-self-mapping. From (v) there exists $\phi_{0} \in \mathscr{R}_{c}$ such that $\alpha\left(\phi_{0}(c), T \phi_{0}\right) \geq 1$. Let, $\left\{\phi_{n}\right\}$ be a sequence in $E_{0}$ such that $\phi_{n} \rightarrow \phi$ as $n \rightarrow \infty$ and $\left(\phi_{n}(c), \phi_{n+1}(c)\right) \in E(G)$ for all $n \in \mathbb{N} \cup\{0\}$. Then, $\alpha\left(\phi_{n}(c), \phi_{n+1}(c)\right) \geq 1$ for all $n \in \mathbb{N} \cup\{0\}$. Thus, from (iv) we get, $\left(\phi_{n}(c), \phi\right) \in E(G)$ for all $n \in \mathbb{N} \cup\{0\}$. That is, $\alpha\left(\phi_{n}(c), \phi\right) \geq 1$ for all $n \in \mathbb{N} \cup\{0\}$. Therefore all conditions of Corollary 13 hold true and $T$ has a PPF dependent fixed point.
Similarly as an application of Corollary 17, we can prove the following Theorem.

Theorem 20. Let $c \in I, S: E_{0} \rightarrow E_{0}, T: E_{0} \rightarrow E$ and $E$ endowed with a graph $G$. Suppose that the following assertions hold true:

(i) there exists $c \in I$ such that $S\left(\mathscr{R}_{c}\right)$ is topologically closed and algebraically closed with respect to difference;

(ii) if $((S \phi)(c),(S \xi)(c)) \in E(G)$, then $(T \phi, T \xi) \in E(G)$;

(iii) assume that

$$
((S \phi)(c),(S \xi)(c)) \in E(G) \Longrightarrow\|T \phi-T \xi\|_{E} \leq \psi(N(\phi, \xi))
$$

for $\phi, \xi \in E_{0}$, where $\psi \in \Psi$;

(iv) if $\left\{S \phi_{n}\right\}$ is a sequence in $E_{0}$ such that $S \phi_{n} \rightarrow S \phi$ as $n \rightarrow \infty$ and $\left(\left(S \phi_{n}\right)(c),\left(S \phi_{n+1}\right)(c)\right) \in E(G)$ for all $n \in$ $\mathbb{N} \cup\{0\}$, then $\left(\left(S \phi_{n}\right)(c), S \phi(c)\right) \in E(G)$ for all $n \in \mathbb{N} \cup\{0\} ;$

(v) there exists $S \phi_{0} \in S\left(\mathscr{R}_{c}\right)$ such that $\left(S \phi_{0}(c), T \phi_{0}\right) \in$ $E(G)$.

\section{Then, $S$ and T have a PPF dependent coincidence point.}

The study of existence of fixed points in partially ordered sets has been initiated by Ran and Reurings [27] with applications to matrix equations. Agarwal et al. [1,2], Ćirić et al. [7], and Hussain et al. [11, 12] presented some new results for nonlinear contractions in partially ordered Banach and metric spaces with applications. Here as an application of our results we deduce some new PPF dependent fixed and coincidence point results whenever the range space is endowed with a partial order.

Definition 21. Let $c \in I, T: E_{0} \rightarrow E$ and $E$ endowed with a partial order $\preceq$. We say that $T$ is a $c$-increasing non-selfmapping if for $\phi, \xi \in E_{0}$ with $\phi(c) \preceq \xi(c)$ we have $T \phi \preceq T \xi$.

Definition 22. Let $c \in I, S: E_{0} \rightarrow E_{0}, T: E_{0} \rightarrow E$ and $E$ endowed with a partial order $\preceq$. We say that the pair $(S, T)$ is $c$-increasing if for $\phi, \xi \in E_{0}$ with $(S \phi)(c) \preceq(S \xi)(c)$ we have $T \phi \preceq T \xi$.

Theorem 23. Let $T: E_{0} \rightarrow E$ and $E$ endowed with a partial order $\preceq$. Suppose that the following assertions holds true:

(i) there exists $c \in I$ such that $\mathscr{R}_{c}$ is topologically closed and algebraically closed with respect to difference;

(ii) $T$ is a c-increasing non-self-mapping;

(iii) Assume that

$$
\|T \phi-T \xi\|_{E} \leq \psi(M(\phi, \xi))
$$

holds for all $\phi, \xi \in E_{0}$ with $\phi(c) \preceq \xi(c)$ where $\psi \in \Psi$;

(iv) if $\left\{\phi_{n}\right\}$ is a sequence in $E_{0}$ such that $\phi_{n} \rightarrow \phi$ as $n \rightarrow \infty$ and $\phi_{n}(c) \preceq \phi_{n+1}(c)$ for all $n \in \mathbb{N} \cup\{0\}$, then $\phi_{n}(c) \preceq$ $\phi(c)$ for all $n \in \mathbb{N} \cup\{0\}$;

(v) there exists $\phi_{0} \in \mathscr{R}_{c}$ such that $\phi_{0}(c) \preceq T \phi_{0}$.

Then, $T$ has a PPF dependent fixed point. 
Proof. Define $\alpha: E \times E \rightarrow[0,+\infty)$ by

$$
\alpha(x, y)= \begin{cases}1, & \text { if } x \preceq y \\ \frac{1}{2} & \text { otherwise. }\end{cases}
$$

First, we prove that $T$ is an $\alpha_{c}$-admissible non-self-mapping. Assume that $\alpha(\phi(c), \xi(c)) \geq 1$. Then, we have $\phi(c) \preceq \xi(c)$. Since $T$ is $c$-increasing, we get $T \phi \preceq T \xi$; that is, $\alpha(T \phi, T \xi) \geq 1$. Thus $T$ is an $\alpha_{c}$-admissible non-self-mapping. From (v) there exists $\phi_{0} \in \mathscr{R}_{c}$ such that $\phi_{0}(c) \preceq T \phi_{0}$. That is, $\alpha\left(\phi_{0}(c), T \phi_{0}\right) \geq$ 1. Let $\left\{\phi_{n}\right\}$ be a sequence in $E_{0}$ such that $\phi_{n} \rightarrow \phi$ as $n \rightarrow \infty$ and $\alpha\left(\phi_{n}(c), \phi_{n+1}(c)\right) \geq 1$ for all $n \in \mathbb{N} \cup\{0\}$. Then, $\phi_{n}(c) \preceq$ $\phi_{n+1}(c)$ for all $n \in \mathbb{N} \cup\{0\}$. Thus, from (iv) we get $\phi_{n}(c) \preceq \phi(c)$ for all $n \in \mathbb{N} \cup\{0\}$. That is, $\alpha\left(\phi_{n}(c), \phi(c)\right) \geq 1$ for all $n \in \mathbb{N} \cup\{0\}$. Therefore all conditions of Corollary 13 hold true and $T$ has a PPF dependent fixed point.

Similarly we can prove following Theorem.

Theorem 24. Let $c \in I, S: E_{0} \rightarrow E_{0}, T: E_{0} \rightarrow E$ and $E$ endowed with a partial order $\preceq$. Suppose that the following assertions hold true:

(i) there exists $c \in I$ such that $S\left(\mathscr{R}_{c}\right)$ is topologically closed and algebraically closed with respect to difference;

(ii) the pair $(S, T)$ is a c-increasing mapping;

(iii) assume that

$$
\|T \phi-T \xi\|_{E} \leq \psi(N(\phi, \xi))
$$

holds for all $\phi, \xi \in E_{0}$ with $(S \phi)(c) \preceq(S \xi)(c)$, where $\psi \in \Psi$;

(iv) if $\left\{S \phi_{n}\right\}$ is a sequence in $E_{0}$ such that $S \phi_{n} \rightarrow S \phi$ as $n \rightarrow \infty$ and $\left(S \phi_{n}\right)(c) \preceq\left(S \phi_{n+1}\right)(c)$ for all $n \in \mathbb{N} \cup\{0\}$, then $\left(S \phi_{n}\right)(c) \preceq S \phi(c)$ for all $n \in \mathbb{N} \cup\{0\}$;

(v) there exists $S \phi_{0} \in S\left(\mathscr{R}_{c}\right)$ such that $S \phi_{0}(c) \preceq T \phi_{0}$.

Then, $S$ and T have a PPF dependent coincidence point.

\section{Further Consequences}

\subsection{Consequences of Corollary 13}

Theorem 25. Let $T: E_{0} \rightarrow E$ and $\alpha: E \times E \rightarrow[0, \infty)$ be two mappings that satisfy the following assertions:

(i) there exists $c \in I$ such that $\mathscr{R}_{c}$ is topologically closed and algebraically closed with respect to difference;

(ii) $T$ is an $\alpha_{c}$-admissible mapping;

(iii) assume that

$$
\alpha(\phi(c), \xi(c))\|T \phi-T \xi\|_{E} \leq \psi(M(\phi, \xi))
$$

holds for all $\phi, \xi \in E_{0}$, where $\psi \in \Psi$;

(iv) if $\left\{\phi_{n}\right\}$ is a sequence in $E_{0}$ such that $\phi_{n} \rightarrow \phi$ as $n \rightarrow \infty$ and $\alpha\left(\phi_{n}(c), \phi_{n+1}(c)\right) \geq 1$ for all $n \in \mathbb{N} \cup\{0\}$, then $\alpha\left(\phi_{n}(c), \phi(c)\right) \geq 1$ for all $n \in \mathbb{N} \cup\{0\} ;$

(v) there exists $\phi_{0} \in \mathscr{R}_{c}$ such that $\alpha\left(\phi_{0}(c), T \phi_{0}\right) \geq 1$.

Then, T has a PPF dependent fixed point.
Proof. Let $\alpha(\phi(c), \xi(c)) \geq 1$. Hence, from (iii) we have

$$
\|T \phi-T \xi\|_{E} \leq \alpha(\phi(c), \xi(c))\|T \phi-T \xi\|_{E} \leq \psi(M(\phi, \xi)) .
$$

That is, all conditions of Corollary 13 are satisfied and $T$ has a PPF dependent fixed point.

Similarly we can prove the following results.

Theorem 26. Let $T: E_{0} \rightarrow E$ and $\alpha: E \times E \rightarrow[0, \infty)$ be two mappings that satisfy the following assertions:

(i) there exists $c \in I$ such that $\mathscr{R}_{c}$ is topologically closed and algebraically closed with respect to difference;

(ii) $T$ is an $\alpha_{c}$-admissible mapping;

(iii) assume that

$$
\left(\|T \phi-T \xi\|_{E}+\epsilon\right)^{\alpha(\phi(c), \xi(c))} \leq \psi(M(\phi, \xi))+\epsilon
$$

holds for all $\phi, \xi \in E_{0}$, where $\epsilon \geq 1$ and $\psi \in \Psi$;

(iv) if $\left\{\phi_{n}\right\}$ is a sequence in $E_{0}$ such that $\phi_{n} \rightarrow \phi$ as $n \rightarrow \infty$ and $\alpha\left(\phi_{n}(c), \phi_{n+1}(c)\right) \geq 1$ for all $n \in \mathbb{N} \cup\{0\}$, then $\alpha\left(\phi_{n}(c), \phi(c)\right) \geq 1$ for all $n \in \mathbb{N} \cup\{0\} ;$

(v) there exists $\phi_{0} \in \mathscr{R}_{c}$ such that $\alpha\left(\phi_{0}(c), T \phi_{0}\right) \geq 1$.

Then, T has a PPF dependent fixed point.

Theorem 27. Let $T: E_{0} \rightarrow E$ and $\alpha: E \times E \rightarrow[0, \infty)$ be two mappings that satisfy the following assertions:

(i) there exists $c \in I$ such that $\mathscr{R}_{c}$ is topologically closed and algebraically closed with respect to difference;

(ii) $T$ is an $\alpha_{c}$-admissible mapping;

(iii) assume that

$$
\left(\alpha(\phi(c), \xi(c))-1+\epsilon^{\prime}\right)^{\|T \phi-T \xi\|_{E}} \leq \epsilon^{\psi(M(\phi, \xi))}
$$

holds for all $\phi, \xi \in E_{0}$, where $1<\epsilon \leq \epsilon^{\prime}$ and $\psi \in \Psi$;

(iv) if $\left\{\phi_{n}\right\}$ is a sequence in $E_{0}$ such that $\phi_{n} \rightarrow \phi$ as $n \rightarrow \infty$ and $\alpha\left(\phi_{n}(c), \phi_{n+1}(c)\right) \geq 1$ for all $n \in \mathbb{N} \cup\{0\}$, then $\alpha\left(\phi_{n}(c), \phi(c)\right) \geq 1$ for all $n \in \mathbb{N} \cup\{0\} ;$

(v) there exists $\phi_{0} \in \mathscr{R}_{c}$ such that $\alpha\left(\phi_{0}(c), T \phi_{0}\right) \geq 1$.

Then, T has a PPF dependent fixed point.

\subsection{Consequences of Corollary 17}

Theorem 28. Let $S: E_{0} \rightarrow E_{0}, T: E_{0} \rightarrow E$ and $\alpha: E \times E \rightarrow$ $[0, \infty)$ be three mappings that satisfy the following assertions:

(i) there exists $c \in I$ such that $S\left(\mathscr{R}_{c}\right)$ is topologically closed and algebraically closed with respect to difference;

(ii) the pair $(S, T)$ is an $\alpha_{c}$-admissible;

(iii) assume that

$$
\alpha((S \phi)(c),(S \xi)(c))\|T \phi-T \xi\|_{E} \leq \psi(N(\phi, \xi))
$$

holds for all $\phi, \xi \in E_{0}$, where $\psi \in \Psi$; 
(iv) if $\left\{S \phi_{n}\right\}$ is a sequence in $E_{0}$ such that $S \phi_{n} \rightarrow S \phi$ as $n \rightarrow \infty$ and $\alpha\left(\left(S \phi_{n}\right)(c),\left(S \phi_{n+1}\right)(c)\right) \geq 1$ for all $n \in$ $\mathbb{N} \cup\{0\}$, then $\alpha\left(\left(S \phi_{n}\right)(c), S \phi(c)\right) \geq 1$ for all $n \in \mathbb{N} \cup\{0\}$;

(v) there exists $S \phi_{0} \in S\left(\mathscr{R}_{c}\right)$ such that $\alpha\left(S \phi_{0}(c), T \phi_{0}\right) \geq 1$.

Then, $S$ and T have a PPF dependent coincidence point.

Theorem 29. Let $S: E_{0} \rightarrow E_{0}, T: E_{0} \rightarrow E$ and $\alpha: E \times E \rightarrow$ $[0, \infty)$ be three mappings that satisfy the following assertions:

(i) there exists $c \in I$ such that $S\left(\mathscr{R}_{c}\right)$ is topologically closed and algebraically closed with respect to difference;

(ii) the pair $(S, T)$ is an $\alpha_{c}$-admissible;

(iii) assume that

$$
\begin{gathered}
\left(\|T \phi-T \xi\|_{E}+\epsilon\right)^{\alpha((S \phi)(c),(S \xi)(c))} \leq \psi(N(\phi, \xi))+\epsilon \\
\text { holds for all } \phi, \xi \in E_{0}, \text { where } \epsilon \geq 1 \text { and } \psi \in \Psi ;
\end{gathered}
$$

(iv) if $\left\{S \phi_{n}\right\}$ is a sequence in $E_{0}$ such that $S \phi_{n} \rightarrow S \phi$ as $n \rightarrow \infty$ and $\alpha\left(\left(S \phi_{n}\right)(c),\left(S \phi_{n+1}\right)(c)\right) \geq 1$ for all $n \in$ $\mathbb{N} \cup\{0\}$, then $\alpha\left(\left(S \phi_{n}\right)(c), S \phi(c)\right) \geq 1$ for all $n \in \mathbb{N} \cup\{0\} ;$

(v) there exists $S \phi_{0} \in S\left(\mathscr{R}_{c}\right)$ such that $\alpha\left(S \phi_{0}(c), T \phi_{0}\right) \geq 1$.

Then, $S$ and T have a PPF dependent coincidence point.

Theorem 30. Let $S: E_{0} \rightarrow E_{0}, T: E_{0} \rightarrow E$ and $\alpha: E \times E \rightarrow$ $[0, \infty)$ be three mappings that satisfy the following assertions:

(i) there exists $c \in I$ such that $S\left(\mathscr{R}_{c}\right)$ is topologically closed and algebraically closed with respect to difference;

(ii) the pair $(S, T)$ is an $\alpha_{c}$-admissible;

(iii) assume that

$$
\left(\alpha((S \phi)(c),(S \xi)(c))-1+\epsilon^{\prime}\right)^{\|T \phi-T \xi\|_{E}} \leq \epsilon^{\psi(N(\phi, \xi))}
$$

holds for all $\phi, \xi \in E_{0}$, where $1<\epsilon \leq \epsilon^{\prime}$ and $\psi \in \Psi$;

(iv) if $\left\{S \phi_{n}\right\}$ is a sequence in $E_{0}$ such that $S \phi_{n} \rightarrow S \phi$ as $n \rightarrow \infty$ and $\alpha\left(\left(S \phi_{n}\right)(c),\left(S \phi_{n+1}\right)(c)\right) \geq 1$ for all $n \in$ $\mathbb{N} \cup\{0\}$, then $\alpha\left(\left(S \phi_{n}\right)(c), S \phi(c)\right) \geq 1$ for all $n \in \mathbb{N} \cup\{0\} ;$

(v) there exists $S \phi_{0} \in S\left(\mathscr{R}_{c}\right)$ such that $\alpha\left(S \phi_{0}(c), T \phi_{0}\right) \geq 1$.

Then, $S$ and T have a PPF dependent coincidence point.

\section{Conflict of Interests}

The authors declare that there is no conflict of interests regarding the publication of this paper.

\section{Acknowledgment}

This article was funded by the Deanship of Scientific Research (DSR), King Abdulaziz University, Jeddah. Therefore, the first author acknowledges with thanks DSR, KAU for financial support.

\section{References}

[1] R. P. Agarwal, M. A. El-Gebeily, and D. O’Regan, "Generalized contractions in partially ordered metric spaces," Applicable Analysis, vol. 87, no. 1, pp. 1-8, 2008.

[2] R. P. Agarwal, N. Hussain, and M. A. Taoudi, "Fixed point theorems in ordered Banach spaces and applications to nonlinear integral equations," Abstract and Applied Analysis, vol. 2012, Article ID 245872, 15 pages, 2012.

[3] M. A. Abbas and T. Nazir, "Common fixed point of a power graphic contraction pair in partial metric spaces endowed with graph," Fixed Point Theory and Applications, vol. 2013, p. 20, 2013.

[4] A. Gh. B. Ahmad, Z. Fadail, H. K. Nashine, Z. Kadelburg, and $S$. Radenovic, "Some new common fixed point results through generalized altering distances on partial metric spaces," Fixed Point Theory and Applications, vol. 2012, p. 120, 2012.

[5] S. R. Bernfeld, V. Lakshmikatham, and Y. M. Reddy, "Fixed point theorems of operators with PPF dependence in Banach spaces," Applicable Analysis, vol. 6, no. 4, pp. 271-280, 1977.

[6] F. Bojor, "Fixed point theorems for Reich type contractions on metric spaces with a graph," Nonlinear Analysis, Theory, Methods and Applications, vol. 75, no. 9, pp. 3895-3901, 2012.

[7] L. Ćirić, M. Abbas, R. Saadati, and N. Hussain, "Common fixed points of almost generalized contractive mappings in ordered metric spaces," Applied Mathematics and Computation, vol. 217, no. 12, pp. 5784-5789, 2011.

[8] L. B. Ćirić, S. M. A. Alsulami, P. Salimi, and P. Vetro, "PPF dependent fixed point results for triangular $\propto_{c}$-admissible mapping," The Scientific World Journal. In press.

[9] B. C. Dhage, "Some basic random fixed point theorems with PPF dependence and functional random differential equations," Differential Equations \& Applications, vol. 4, pp. 181-195, 2012.

[10] M. Geraghty, "On contractive mappings," Proceedings of the American Mathematical Society, vol. 40, pp. 604-608, 1973.

[11] N. Hussain, S. Al-Mezel, and P. Salimi, "Fixed points for $\psi$ graphic contractions with application to integral equations," Abstract and Applied Analysis, vol. 2013, Article ID 575869, 11 pages, 2013.

[12] N. Hussain, A. R. Khan, and R. P. Agarwal, "Krasnosel'skii and Ky Fan type fixed point theorems in ordered Banach spaces," Journal of Nonlinear and Convex Analysis, vol. 11, no. 3, pp. 475489, 2010.

[13] M. A. Kutbi, N. Hussain, and P. Salimi, "Best proximity point results for modified $\alpha$ - $\psi$-proximal rational contractions," Abstract and Applied Analysis, vol. 2013, Article ID 927457, 14 pages, 2013.

[14] N. Hussain, P. Salimi, and A. Latif, "Fixed point results for single and set-valued $\alpha-\eta-\psi$-contractive mappings," Fixed Point Theory and Applications, vol. 2013, p. 212, 2013.

[15] J. Jachymski, "The contraction principle for mappings on a metric space with a graph," Proceedings of the American Mathematical Society, vol. 136, no. 4, pp. 1359-1373, 2008.

[16] R. Johnsonbaugh, Discrete Mathematics, Prentice-Hall, New Jersey, NJ, USA, 1997.

[17] A. Kaewcharoen, "PPF dependent common fixed point theorems for mappings in Bnach spaces," Journal of Inequalities and Applications, vol. 2013, p. 287, 2013.

[18] E. Karapinar, P. Kumam, and P. Salimi, "On $\alpha-\psi$-Meir-Keeler contractive mappings," Fixed Point Theory and Applications, vol. 2013, p. 94, 2013. 
[19] E. Karapinar and B. Samet, "Generalized $(\alpha-\psi)$ contractive type mappings and related fixed point theorems with applications," Abstract and Applied Analysis, vol. 2012, Article ID 793486, 17 pages, 2012.

[20] F. Khojasteh, E. Karapinar, and S. Radenovic, " $\theta$-metric space: a generalization," Mathematical Problems in Engineering, vol. 2013, Article ID 504609, 7 pages, 2013.

[21] M. Mursaleen, S. Mohiuddine, and R. P. Agarwal, "Coupled fixed point theorems for $\alpha$ - $\psi$-contractive type mappings in partially ordered metric spaces," Fixed Point Theory and Applications, vol. 2012, p. 228, 2012.

[22] B. Samet, C. Vetro, and P. Vetro, "Fixed point theorems for $\alpha$ - $\psi$-contractive type mappings," Nonlinear Analysis, Theory, Methods and Applications, vol. 75, no. 4, pp. 2154-2165, 2012.

[23] M. Samreen and T. Kamran, "Fixed point theorems for integral G-contraction," Fixed Point Theory and Applications, vol. 2013, p. 149, 2013

[24] P. Salimi, A. Latif, and N. Hussain, "Modified $\alpha-\psi$-contractive mappings with applications," Fixed Point Theory and Applications, vol. 2013, p. 151, 2013.

[25] P. Salimi, C. Vetro, and P. Vetro, "Some new fixed point results in non-Archimedean fuzzy metric spaces," Nonlinear Analysis: Modelling and Control, vol. 18, no. 3, pp. 344-358, 2013.

[26] P. Salimi, C. Vetro, and P. Vetro, "Fixed point theorems for twisted $(\alpha, \beta)-\psi$-contractive type mappings and applications," Filomat, vol. 27, no. 4, pp. 605-615, 2013.

[27] A. C. M. Ran and M. C. B. Reurings, "A fixed point theorem in partially ordered sets and some applications to matrix equations," Proceedings of the American Mathematical Society, vol. 132, no. 5, pp. 1435-1443, 2004.

[28] N. Hussain, M. H. Shah, and M. A. Kutbi, "Coupled coincidence point theorems for nonlinear contractions in partially ordered quasi-metric spaces with a Q-function," Fixed Point Theory and Applications, vol. 2011, Article ID 703938, 21 pages, 2011.

[29] Y. J. Cho, M. H. Shah, and N. Hussain, "Coupled fixed points of weakly F-contractive mappings in topological spaces," Applied Mathematics Letters, vol. 24, no. 7, pp. 1185-1190, 2011.

[30] M. A. Kutbi, J. Ahmad, and A. Azam, "On fixed points of $\alpha$ - $\psi$-contractive multi-valued mappings in cone metric spaces," Abstract and Applied Analysis, vol. 2013, Article ID 313782, 6 pages, 2013.

[31] R. P. Agarwal, P. Kumam, and W. Sintunavarat, "PPF dependent fixed point theorems for an $\alpha_{c}$-admissible non-self mapping in the Razumikhin class," Fixed Point Theory and Applications, vol. 2013, article 280, 2013. 


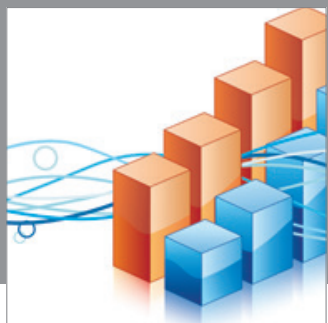

Advances in

Operations Research

mansans

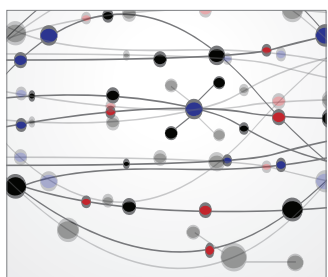

The Scientific World Journal
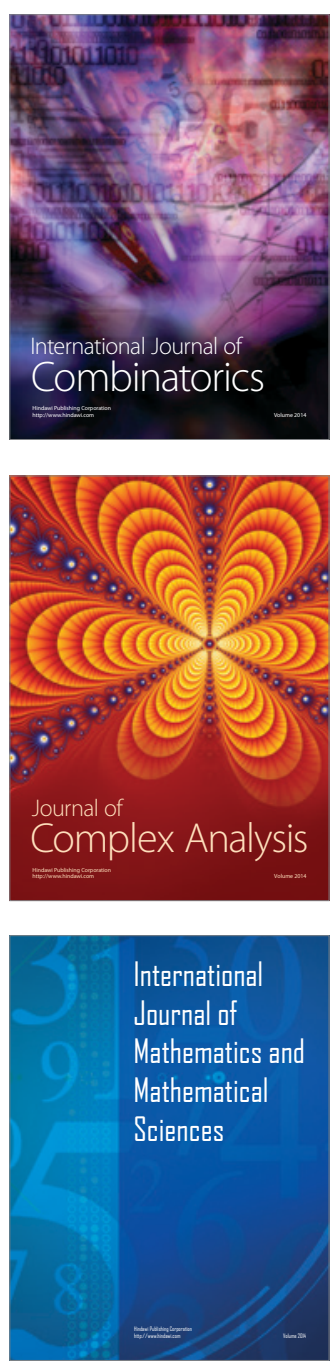
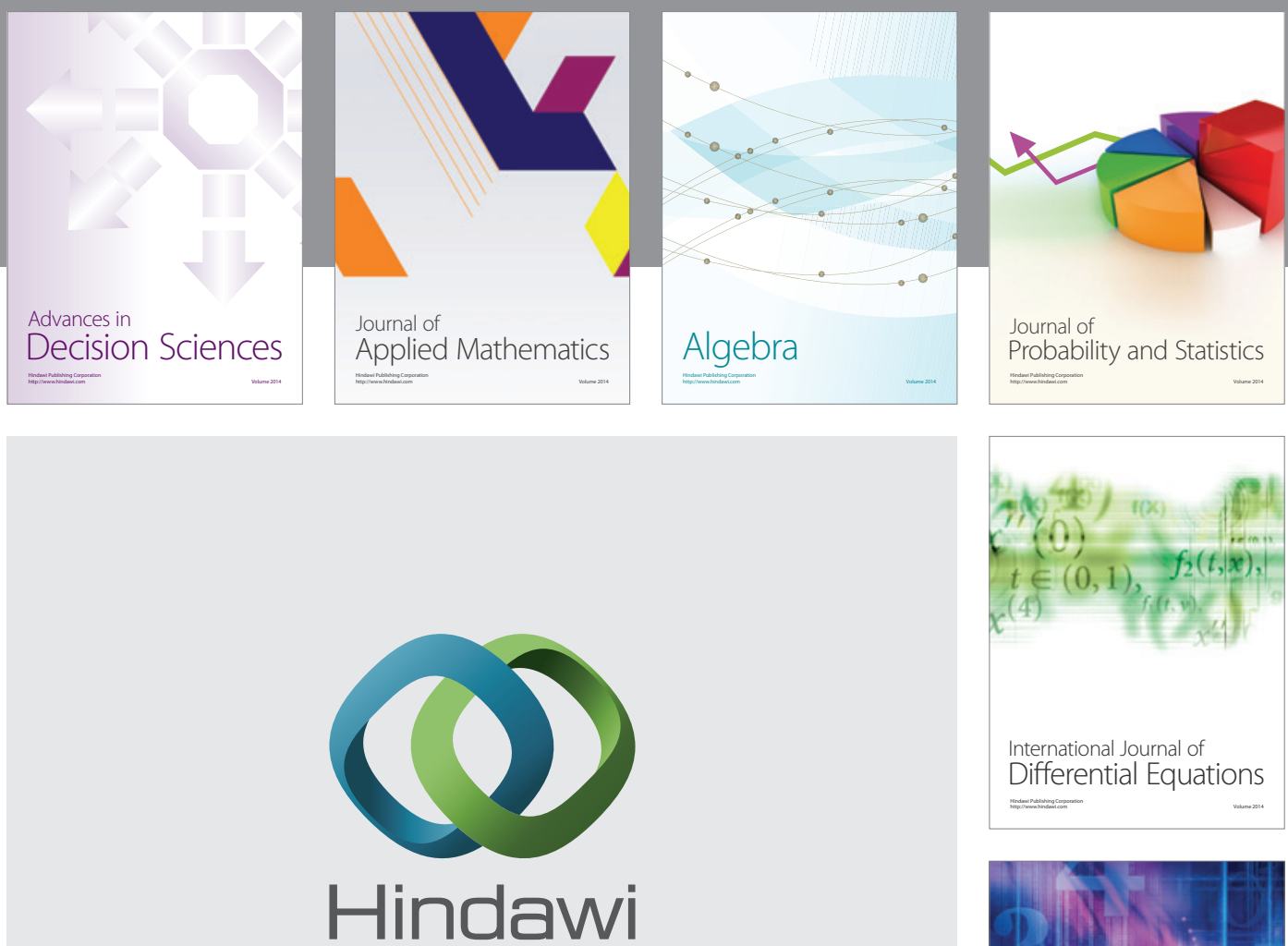

Submit your manuscripts at http://www.hindawi.com
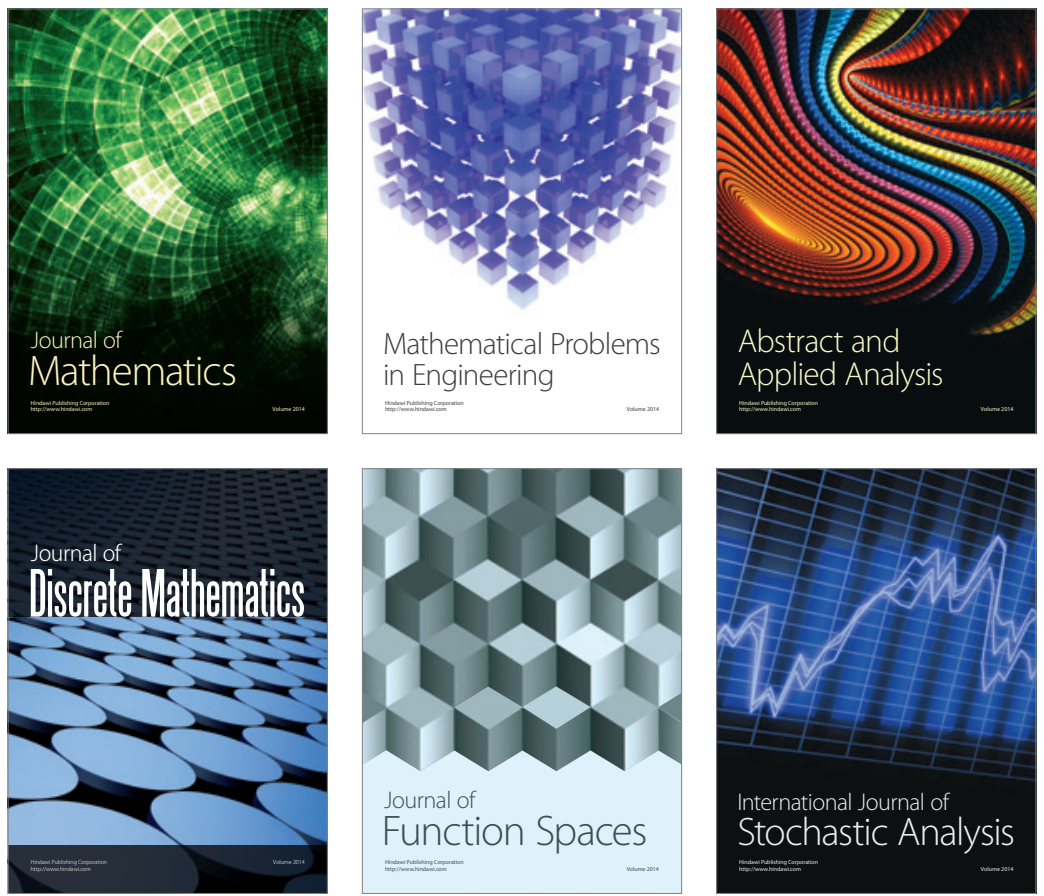

Journal of

Function Spaces

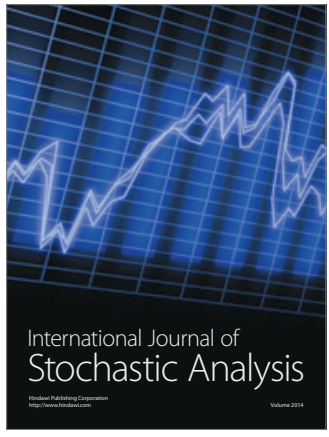

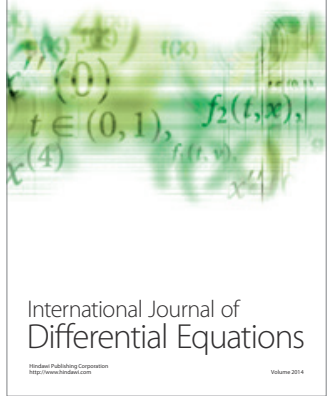
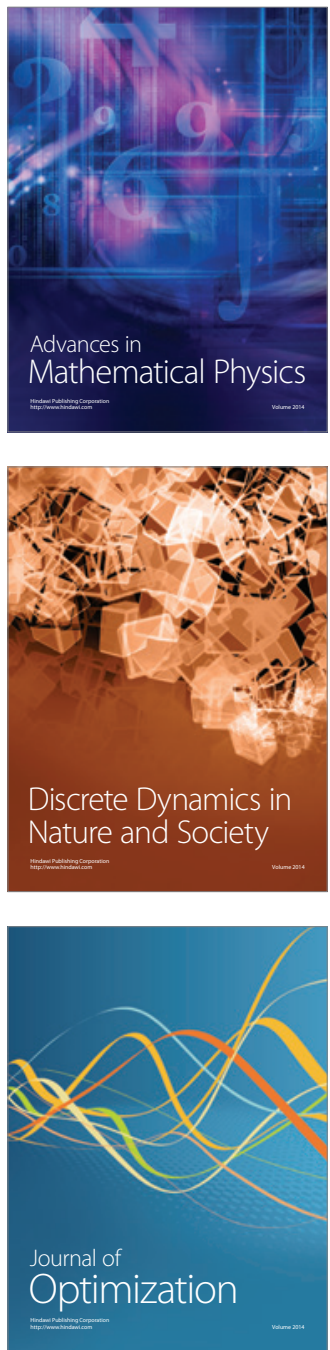